\title{
Novo Método para Tratamento da Angiodisplasia de Cólon
}

\author{
New Method of Treating Colonic Angiodysplasia
}

\author{
ANTÔNIO HILÁRIO ALVES FREITAS - TSBCP ${ }^{1}$, DERIVAL NELMO DOS SANTOS ${ }^{1}$, CÁSSIADE MELOTODESCHI ${ }^{1}$, \\ SÉRGIOAPARECIDO VITAL ${ }^{1}$, KANTHYAARREGUY DE SENABORGES ${ }^{1}$, PATRÍCIARIBEIRODE BABO ${ }^{2}$
}

${ }^{1}$ Oficiais Médicos do Serviço de Endoscopia Digestiva; ${ }^{2}$ Médica Residente do Serviço de Endoscopia Digestiva.

\begin{abstract}
FREITAS AHA; SANTOS DN; TODESCHI CM; VITAL SA; BORGES KAS; BABO PR. Novo Método para Tratamento da Angiodisplasia de Cólon. Rev bras Coloproct, 2009;29(4): 485-488.

RESUMO: A utilização da colonoscopia como método de diagnóstico e tratamento possibilitou avanços na abordagem da angiodisplasia de cólon, anomalia vascular responsável por um terço das hemorragias gastrointestinais baixas. Trata-se de paciente com 65 anos, insuficiência renal crônica dialítica e coronariopata grave, apresentando episódios de hematoquezia volumosa ao iniciar uso de agregantes plaquetários, necessitando múltiplas hemotransfusões. Colonoscopia diagnosticou lesões vasculares no ceco, onde existe grande risco de perfuração durante intervenções terapêuticas. Há descrições na literatura do uso de agentes hemostáticos tais como plasma de Argônio e vasoconstrictores, aplicados separadamente. Foi realizado tratamento endoscópico das angiodisplasias com aplicação de Argônio, após elevação das lesões com soro fisiológico, e pela primeira vez descrita no Brasil, acrescentado vasoconstrictor à solução.
\end{abstract}

Descritores: Colonoscopia. Angiodisplasia. Cólon. Argônio.

\section{INTRODUÇÃO}

As anomalias vasculares de cólon são causas freqüentes de hemorragia digestiva baixa ${ }^{1}$.

A colonoscopia utilizada como método terapêutico vem alcançando bons resultados tanto em neoplasias, quanto nos sangramentos do trato gastrointestinal $^{2}$.

O caso descrito é de um paciente com 65 anos de idade, comorbidades graves, apresentando episódios de hematoquezia volumosa ao iniciar uso de antiagregantes plaquetários. Colonoscopia diagnosticou lesões vasculares aracneiformes com sinais de sangramento, localizadas no ceco.

O tratamento endoscópico das anomalias vasculares do cólon, com hemostasia utilizando soluções (soro fisiológico, esclerosantes) ou métodos térmicos (cautérios, Argônio) já foi descrito anteriormente no Brasil. No presente trabalho, foi adicionado vasoconstrictor à solução no intuito de estender o tempo de elevação das lesões e aumentar a segurança durante a aplicação do Argônio (primeiro relato nacional desta associação de agentes hemostáticos no intestino grosso).

\section{RELATO DE CASO}

Trata-se de paciente com 65 anos, sexo masculino, tabagista, hipertenso, portador de insuficiência renal crônica dialítica e coronariopatia grave.

A possibilidade de transplante renal somente poderia ser avaliada após realização de angioplastia coronariana. No entanto, apresentava episódios de hematoquezia volumosa ao iniciar uso de antiagregantes plaquetários, essenciais após intervenções hemodinâmicas.

Internado no Hospital da Polícia Militar de Minas Gerais com quadro de hematoquezia volumosa e anemia. A endoscopia digestiva alta sem alterações. $\mathrm{O}$ exame proctológico com retossigmoidoscopia mostrou grande volume de sangue no reto. A colonoscopia diagnosticou lesões angiodisplásicas no ceco. Visando

Trabalho realizado no Hospital da Polícia Militar de Minas Gerais. 
terapêutica futura da coronariopatia e o consequiente uso de antiagregantes plaquetários, o paciente foi encaminhado para terapêutica das lesões vasculares do cólon.

Foi realizado tratamento endoscópico de duas angiodisplasias localizadas no ceco, elevando-as com solução fisiológica acrescida de vasoconstrictor (adrenalina 1:200.000), 3 a 5 ml injetados com catéter endoscópico, adjacente às anomalias, e a seguir, empregado plasma de Argônio (ARGON 4 - WEM Equipamentos Elétricos LTDA - Ribeirão Preto / S.P.) utilizando corrente de $40 \mathrm{~W}$ e fluxo de gás de $3 \mathrm{~L}$, em pulsos repetidos de 1 segundo de duração, até completa fulguração dos vasos.

Ato sem intercorrências, paciente apresentando boa evolução, recebendo alta hospitalar no dia seguinte, sem manifestação clínica ou laboratorial de ressangramento e com melhora da anemia durante os 90 dias subseqüentes, momento desta publicação.

\section{DISCUSSÃO}

As anomalias vasculares do cólon são causas freqüentes de sangramento gastrointestinal, responsáveis por um terço das hemorragias gastrointestinais baixas ${ }^{1}$. Predominam na população entre 60-70 anos de idade e acometem igualmente ambos os sexos. Constitui achado incidental na maioria dos casos, e apenas merecem ser tratadas quando maiores que 8 $\mathrm{mm}$ e/ou apresentarem sinais de sangramento. Quando são causas de sangramento e não recebem tratamento, $85 \%$ ressangram nos próximos 20 meses ${ }^{2}$. Em sua grande maioria localizam-se nos segmentos proximais do cólon, e situam-se na camada submucosa. Em pacientes com insuficiência renal, é a principal causa de sangramento intestinal baixo recorrente ${ }^{3}$.

A colonoscopia é o melhor método para diagnóstico de anomalias vasculares de cólon. O avanço na terapêutica endoscópica possibilitou abordagem da angiodisplasia intestinal. Vários métodos têm sido utilizados para hemostasia, tais como: injeção de soluções na submucosa (soro fisiológico, esclerosantes, vasoconstrictores); e métodos térmicos (cautérios monopolar e bipolar, gás Argônio) 4, 5, 6, 7, 8,9.

A predominância das lesões no cólon direito significa risco maior de complicações no tratamento das anomalias vasculares. A parede do intestino neste segmento é muito fina e perfura-se mais facilmente, principalmente quando se utiliza método térmico para alcançar a camada submucosa que requer aplicação de maior quantidade de energia.

Anteriormente eram utilizadas soluções esclerosantes e vasoconstrictores injetados junto às lesões, obtendo-se sucesso, mas com grande índice de recorrência. Com a incorporação do Argônio a partir da década de 90, tornou-se o método de escolha para o tratamento das anomalias vasculares do sistema digestivo $10,11,12,13,14,15,16$.

O plasma de Argônio, por não ser método de contato direto, tem sido o muito utilizado no tratamento de lesões do trato gastrointestinal (esôfago de Barret, varizes, pólipos). Nas patologias gastroesofágicas apresenta-se como alternativa segura, já que tais segmentos possuem parede muito espessa, com risco menor de perfuração, apesar de alguns casos descritos ${ }^{16}$. No intestino grosso, principalmente em cólon direito, utiliza-se como artifício a confecção de uma bolha de solução fisiológica junto à lesão vascular para aplicação do Argônio (Figura 1).

Trabalhos experimentais em suínos mostram que as lesões térmicas da camada muscular circular do intestino grosso caem de $90 \%$ para $10 \%$, e da camada longitudinal de $50 \%$ para $0 \%$, quando se utiliza a bolha de solução salina antes da aplicação do Argônio ${ }^{17}$.

O método aplicado neste trabalho foi descrito por um grupo de autores do Reino Unido, mas é iné-

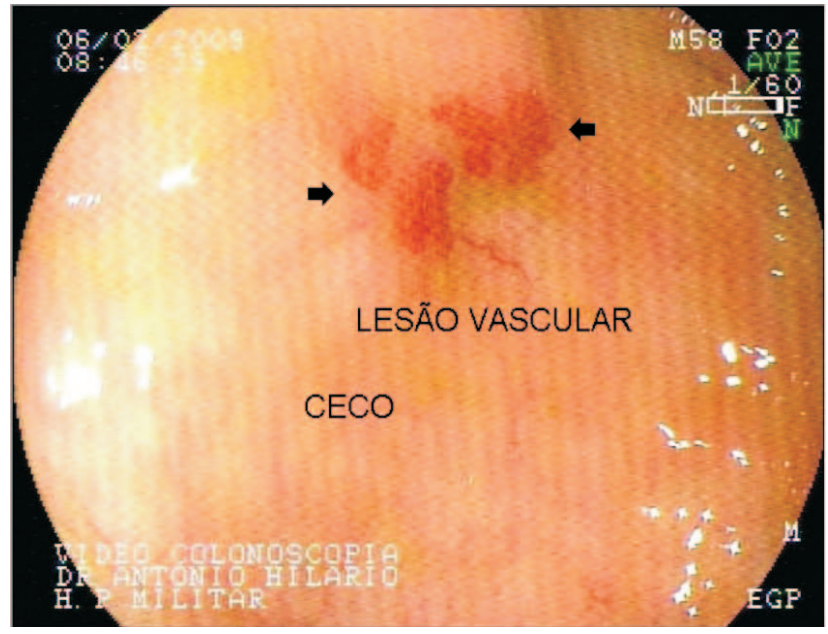

Figura 1 - Bolha contendo solução de vasoconstrictor, aplicada próximo à anomalia vascular, no ceco. 
dito no Brasil ${ }^{18}$. Até o momento, o gás Argônio era aplicado diretamente na lesão ou fazia-se a bolha com solução fisiológica. A inovação vem com a associação de soro fisiológico e vasoconstrictor (solução de adrenalina 1:200.000) aplicados na submucosa. O principal benefício é que o efeito somatório do vasoconstrictor aumenta o tempo de permanência da bolha e impede o sangramento durante a utilização do Argônio, que pode então ser aplicado com maior precisão (Figura 2).

Conclui-se que esta associação tática é factível, possui ampla aplicação prática, e pode ser somada ao arsenal do especialista que realiza a colonoscopia terapêutica.

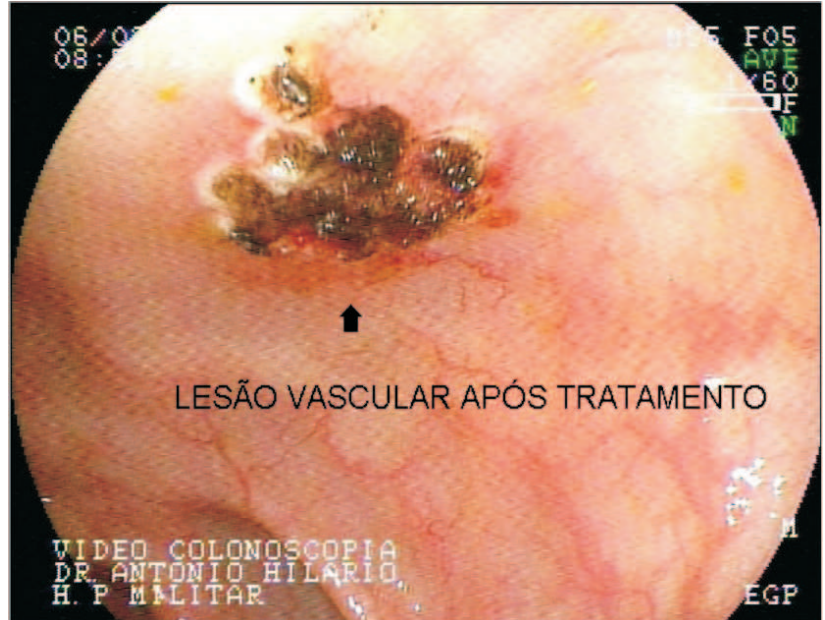

Figura 2 - Local após aplicação de vasoconstrictor e plasma de Argônio.

\begin{abstract}
The utilization of colonoscopy as a diagnostic and treatment tool allowed for advances in the approach of colonic angiodysplasia. This entity is responsible for one third of the cases of lower gastrointestinal bleeding. We discuss a 65 year old patient who suffered from coronary heart disease and chronic renal insufficiency that required dialysis. This patient developed massive hematochezia after starting the use of platelet aggregation inhibitors and required multiple transfusions. The colonoscopy identified vascular lesions in the cecum, where exist a high risk of perforation during therapeutics interventions. There are descriptions in medical literature of the use of hemostatic agents such as argon plasma coagulation and vasoconstrictors used separately. The angiodysplasia was treated endoscopically by the application of argon plasma laser after elevating the lesions with saline and, described for the first time in Brazil, adding vasoconstrictor to the saline solution.
\end{abstract}

Key words: Colonoscopy; Angiodysplasia; Colon; Argon.

\section{REFERÊNCIAS}

1. Browder W, Cerise EJ, Litwin MS. Impact of emergency angiography in massive lower gastrointestinal bleeding. Ann Surg 1986; 204:530-6.

2. Foutch PG, Rex DK, Lieberman DA. Prevalence and natural history of colonic angiodysplasia among healthy asymptomatic people. Am J Gastroenterol 1995; 90:564-7.

3. Saudi J. Gastrointestinal angiodisplasia in chronic renal failure. Kidney Dis Transpl 2008; 19:809-12.

4. Bianco MA, Rotondano G, Marmo R, et al. Combined epinephrine and bipolar probe coagulation vs. bipolar probe coagulation alone for bleeding peptic ulcer: a randomized, controlled trial. Gastrointest Endosc 2004;60:910-5.

5. Chau CH, Siu WT, Law BK, et al. Randomized controlled trial comparing epinephrine injection plus heat probe coagulation versus epinephrine injection plus argon plasma coagulation for bleeding peptic ulcers. Gastrointest Endosc 2003;57:45561.

6. Gevers AM, De Goede E, Simoens M, et al. A randomized trial comparing injection therapy with hemoclip and with injection combined with hemoclip for bleeding ulcers. Gastrointest Endosc 2002;55:466-9.

7. Jensen DM, Cheng S, Kovacs TO, et al. A controlled study of ranitidine for the prevention of recurrent hemorrhage from duodenal ulcer. N Engl J Med 1994;330:382-6.

8. Lin HJ, Hsieh YH, Tseng GY, et al. A prospective, randomized trial of large- versus small-volume endoscopic injection of epinephrine for peptic ulcer bleeding. Gastrointest Endosc 2002;55:615-9.

9. Wilcox CM, Canakis J, Monkemuller KE, et al. Patterns of bleeding after endoscopic sphincterotomy, the subsequent risk of bleeding, and the role of epinephrine injection. Am J Gastroenterol 2004;99:244-8.

10. Basu KK, Pick B, Bale R, et al. Efficacy and one year follow up of argon plasma coagulation therapy for ablation of Barrett's oesophagus: factors determining persistence and recurrence of Barrett's epithelium. Gut 2002;51:776-80.

11. Brooker JC, Saunders BP, Shah SG, et al. Treatment with argon plasma coagulation reduces recurrence after piecemeal resection of large sessile colonic polyps: a randomized trial and recommendations. Gastrointest Endosc 2002;55:371-5. 
12. Nakamura S, Mitsunaga A, Murata Y, et al. Endoscopic induction of mucosal fibrosis by argon plasma coagulation (APC) for esophageal varices: a prospective randomized trial of ligation plus APC vs. ligation alone. Endoscopy 2001;33:210-5.

13. Schulz H, Miehlke S, Antos D, et al. Ablation of Barrett's epithelium by endoscopic argon plasma coagulation in combination with high-dose omeprazole. Gastrointest Endosc 2000;51:659-63.

14. Skok P, Krizman I, Skok M. Argon plasma coagulation versus injection sclerotherapy in peptic ulcer hemorrhage: a prospective, controlled study. Hepatogastroenterology 2004;51:165-70.

15. Zlatanic J, Waye JD, Kim PS, et al. Large sessile colonic adenomas: use of argon plasma coagulator to supplement piecemeal snare polypectomy. Gastrointest Endosc 1999;49:731-5.
16. Wahab PJ, Mulder CJ, den Hartog G, et al. Argon plasma coagulation in flexible gastrointestinal endoscopy: pilot experiences. Endoscopy 1997;29:176-81.

17. Norton ID, Wang L, Levine SA, et al. Efficacy of colonic submucosal saline solution injection for the reduction of iatrogenic thermal injury. Gastrointest Endosc 2002;56:95-9.

18. Suzuki N, Arebi N, Saunders BP. A novel method of treating colonic angiodisplasia. Gastrointestinal Endoscopy 2006; 64:424-427.

\section{Endereço para correspondência:} ANTÔNIO HILÁRIO ALVES FREITAS

Rua Paracatu, 838 / 206 - Barro Preto 30180.090 - Belo Horizonte - Minas Gerais Telefone e FAX: 31-3337.9988 Celular: 31-9956.1363

E-mail: hilariofreitas@ig.com.br 